\title{
Emerging and Experimental Agents for Anal Cancer: What is New?
}

This article was published in the following Dove Press journal:

Journal of Experimental Pharmacology

João Paulo F Farias'

Maria Helena C Rangel da

Silva ${ }^{2}$

Alexandre A Jácome $\mathbb{D}^{2}$

'Department of Gastrointestinal Medical Oncology, Oncoclínicas, Rio de Janeiro, Brazil; ${ }^{2}$ Department of Gastrointestinal Medical Oncology, Oncoclínicas, Belo Horizonte, Brazil
Correspondence: Alexandre A Jácome Department of Gastrointestinal Medical Oncology, Oncoclinicas, Rua Roma 56I, Belo Horizonte, MG, 30360-680, Brazil Tel +55 3l 2/26-8600

Email alexandre.jacome@medicos. oncoclinicas.com

\begin{abstract}
Squamous cell carcinoma of the anal canal (SCCA) is an HPV-related malignancy with rising incidence in the past few decades in the US, characterized by high rates of complete response to chemoradiotherapy with curative intent. However, in a long-term follow-up, a meaningful subgroup of patients with locally advanced disease presents disease recurrence, which demands treatments with high morbidity and important impact in the quality of life. In metastatic or unresectable disease, palliative chemotherapy is the standard of care, but it is still associated with a dismal prognosis. Novel agents are urgently needed in the systemic therapy of SCCA. From a translational standpoint, there are many hurdles to overcome, since PI3KCA mutation is the most frequent genetic abnormality and actionable mutations are rarely found in SCCA, as well as it is characterized by low tumor mutational burden and low rates of high-frequency microsatellite instability. But the latest studies of immunotherapeutic approaches have produced promising findings and this therapeutic strategy is the major path being followed in the ongoing clinical trials. The latest advances in the systemic therapy of SCCA have provided the framework for the conception of new clinical trials. Therefore, carboplatin plus paclitaxel have become the backbone for novel agents. Immune checkpoint inhibitors (ICIs), mainly anti-PD-1 monoclonal antibodies, such as retifanlimab, nivolumab, and atezolizumab have been studied in Phase III trials with chemotherapy in first-line therapy. Likewise, ICIs have been evaluated in locally advanced and refractory disease. Novel technologies, such as bispecific antibodies, and immunotherapeutic approaches, such as vaccines and adoptive T-cell therapies, have also been tested in ongoing clinical trials. Immunotherapy may bring practice-changing advances in the systemic therapy of SCCA in the next few years and it might play a larger role in the therapeutic management of this challenging disease.
\end{abstract}

Keywords: chemotherapy, molecular targeted therapy, immunotherapy, monoclonal antibodies, angiogenesis inhibitors

\section{Introduction}

Squamous cell carcinoma of the anal canal (SCCA) is an orphan disease, responsible for approximately $2.5 \%$ of all gastrointestinal malignancies. ${ }^{1}$ Nevertheless, its incidence rates have been steadily increasing in the past decade, accounting for more than 8500 new cases yearly in the United States. ${ }^{1-3}$ Globally, country-specific epidemiological patterns have been observed, but rising incidence rates of the disease have also been described in several high-income countries, such as Australia, Canada, Denmark, France, Italy, Netherlands, and the UK. ${ }^{4}$ It is etiologically linked to human papillomavirus (HPV) infection and presents higher incidence rates in immunocompromised patients, especially those infected by HIV. 
Chemoradiotherapy (CRT) is a highly effective treatment for localized SCCA, associated with complete response (CR) rates in approximately $90 \%$ of the patients. ${ }^{5,6}$ The combination of infusional 5 -fluorouracil (5-FU) or capecitabine with mitomycin concurrent with $50-54$ Gy of radiation therapy has remained as the standard of care of localized disease since the advent of Nigro regimen. ${ }^{5-7}$ However, despite improvements in the radiation therapy techniques, including the incorporation of intensity-modulated radiation therapy (IMRT), it is estimated that $20 \%$ to $44 \%$ of SCCA patients will present disease recurrence in a long-term follow-up, mainly those with T3/T4 and/or node-positive disease. ${ }^{5,6,8-11}$ Disease recurrence is associated with substantial morbidity and mortality. Local recurrence may be managed with salvage surgery, which has a negative impact in the quality of life, and systemic recurrence has a grim prognosis, with an estimated median overall survival (OS) ranging from 12 to 20 months. ${ }^{12}$

Systemic therapy of SCCA is an unmet clinical need. The first randomized clinical trial in advanced disease was published in 2020, and it demonstrated that carboplatin plus paclitaxel should be the standard of care in first-line setting, since it showed superior OS and a more favorable safety profile compared with cisplatin plus 5-FU. ${ }^{12}$ The development of clinical trials and targeted therapies in such an orphan disease is challenging. Immunotherapeutic approaches, including the use of immune checkpoint inhibitors (ICIs), has demonstrated promising results in refractory metastatic disease, ${ }^{13,14}$ but new therapeutic strategies are urgently needed in the management of patients with recurrent and/or metastatic disease.

In this review, we intend to present the main strategies being explored in the therapeutic development of the systemic therapy of SCCA, the ongoing clinical trials, and the perspectives for the management of this complex disease.

\section{Standard of Care}

CRT has remained as the standard of care of locally advanced SCCA for nearly five decades. ${ }^{7}$ The Nigro regimen is composed of infusional $5-\mathrm{FU} 1000 \mathrm{mg} / \mathrm{m}^{2}$ on days 1 to 4 and 29 to 32 , plus mitomycin $10 \mathrm{mg} / \mathrm{m}^{2}$ on day 1 , concurrent with $50-54 \mathrm{~Gy}$ of radiation therapy (RT). ${ }^{7}$ T1N0 tumors with well or moderately differentiated histology may be considered to excision alone, since there is adequate margins and sphincter preservation. ${ }^{15,16} \mathrm{NCCN}$ Guidelines suggest that local excision upfront should be reserved to superficially invasive anal cancers, defined as completely excised lesion with less than $4 \mathrm{~mm}$ of basement membrane invasion and a maximal horizontal spread of less than $8 \mathrm{~mm} .{ }^{15}$

5-FU plus mitomycin is the regimen of choice with concurrent RT, but the combination of 5-FU plus cisplatin has demonstrated comparable efficacy. ${ }^{6,11}$ Based on the phase III RTOG 98-11 trial, 5-FU plus mitomycin was associated with significantly higher disease-free survival (DFS) (68\% versus $58 \%)$ and OS (78\% versus $71 \%$ ) compared with 5 -FU plus cisplatin. ${ }^{6,11}$ On the other hand, another phase III trial, ACT II, showed no statistically significant difference between the two regimens in CR rates at 6 months $(90.5 \%$ vs $89.6 \%$ with mitomycin versus cisplatin, respectively), colostomyfree survival (CFS), DFS, and OS. ${ }^{5}$ There was no statistical difference in the toxicities of the two regimens, with the exception of higher hematological toxicity in the mitomycin arm.

ACT II trial also demonstrated that the timing of achievement of complete clinical response is later than the historically recommended $6-8$ weeks. The decision to perform abdominoperineal resection as surgical salvage for persistent disease should not be made before 26 weeks, given the typical slow regression of the SCCA following RT and the ability to still achieve a complete response. ${ }^{5}$ A biopsy of the anal canal for assessment of complete response is discouraged due to the high risk of ulceration and risk of poor wound healing following completion of RT.

For patients with metastatic disease, systemic chemotherapy remains the standard treatment. Based on the randomized Phase II InterAACT trial, carboplatin plus paclitaxel present higher OS (20.0 versus 12.3 months, $\mathrm{p}=0.014$ ) and lower toxicity rates compared to 5 -FU plus cisplatin. Recent single-arm phase II study also suggests high therapeutic activity ( $89 \%$ of objective response) of the triplet regimen DCF (docetaxel plus cisplatin plus 5-FU) in first-line therapy of metastatic SCCA. However, owing to the high toxicity rates, this combination should be reserved to fit patients who need more intensive therapy. ${ }^{17}$

There is no standard therapy for patients who fail to first-line systemic therapy. If not previously used, the combination of 5-FU plus cisplatin may be considered. Recent single-arm phase II trials demonstrated promising findings of ICIs in refractory patients. Nivolumab reached 
an overall response rate (ORR) of $24 \%$ and pembrolizumab $12 \%$. $^{13,14}$

\section{Molecular Characterization of SCCA}

Some of the molecular abnormalities associated with the malignant transformation triggered by HPV infection in epithelial cells are common to all HPV-related malignancies, irrespective of the primary site, but cervical cancer, HPVpositive head and neck cancer, and SCCA have specific genomic and epigenomic alterations that must be unveiled for a more rationale therapeutic development in these tumors.

The description of the tumor microenvironment in SCCA, such as PD-L1 expression, as well as the identification of potential prognostic and predictive biomarkers, and the characterization of the epigenomic, genomic and transcriptomic abnormalities are paramount for the conception of clinical trials addressing novel therapies, such as immunotherapy and targeted therapy. ${ }^{18}$

The completion of comprehensive genomic profiling studies in rare tumors is arduous, but recent studies have provided pivotal data. PIK3CA gene mutation is the most frequent genetic abnormality in SCCA, identified in $32 \%$ to $88 \%$ of the tumors. ${ }^{19-22}$ Genes important in histone modification, such as MLL3 and MLL2, were also found frequently mutated, as well as genes important to DNA damage repair (p53, ATM, BRCA 1, BRCA 2), chromatin remodeling (EP300, SMARCB1, SMARCA4), and activation of $\mathrm{Wnt} / \beta$ catenin signaling (FAM123B). ${ }^{19}$

It is also suggested that SCCA has a low tumor mutational burden (TMB), with a mean number of 2.5-3.5 somatic mutations $/ \mathrm{Mb}$, similar to those identified in other HPV-related malignancies. ${ }^{20}$ It seems that TMB is low even in the uncommon HPV-negative SCCA, which is associated with a higher probability of p53 mutation. ${ }^{20}$

Interestingly, clinically relevant genomic alterations such as KRAS, NRAS, BRAF, EGFR and HER2 are rare in SCCA. ${ }^{19,20,22}$ Additionally, DNA methylation status seems to differ according to tumor volume, which raises the hypothesis of a potential role of epigenetic events in the progression of the disease. ${ }^{18,21}$

\section{Therapeutic Agents}

\section{Immunotherapeutic Approaches} Immune Checkpoint Inhibitors (ICls)

HPV infection is associated with a pro-tumorigenic and immunosuppressive local microenvironment, which also presents accumulation of PD-L1-expressing M2 macrophages, contributing to the suppression of cytotoxic T-cell responses. ${ }^{19,20,23-26}$ Nevertheless, the efficacy of immunotherapy, mainly of ICIs, has been modest in an unselected population of patients with SCCA. The reasons to explain the moderate efficacy are not clear, but they may be associated to low TMB and to low rates of highfrequency microsatellite instability (MSI-H) found in anal tumors, as aforementioned. Correlative studies from Epitopes-HPV01 and 02 trials have demonstrated that SCCA patients exposed to regimen composed of docetaxel, cisplatin, and 5-fluorouracil (DCF) showed enhanced anti-telomerase immunity, and that high levels of monocytic-myeloid-derived-suppressor cells (MDSC) were associated with poorer prognosis. ${ }^{27}$ Anti-telomerase CD4+ Th1-immunity and MDSC may also be implicated in the sensitivity to ICIs. Nevertheless, a small subgroup of SCCA patients seems to be especially sensitive to immunotherapeutic approaches.

The first study to explore such potential was the phase II NCI9673, which evaluated 37 refractory patients with advanced SCCA, irrespective of PD-L1 expression. ${ }^{13}$ With a median follow-up of 10.1 months, the use of nivolumab $3 \mathrm{mg} / \mathrm{kg}$ each two weeks was associated with an overall response rate (ORR) of $24 \%$. Two patients reached CR, with duration of response longer than 24 months in one of them. In addition, seventeen patients showed stable disease, with an overall disease control rate (DCR) of $72 \%$, and a median duration of treatment of 5.8 months. Anemia, fatigue, rash, and hypothyroidism were the grade 3 adverse events described, with a rate of $14 \%$. Correlative studies demonstrated that responder patients had higher baseline percentages of $\mathrm{CD} 8+\mathrm{T}$ cells and Granzyme B. Among the patients with CD8+ T cells, responders also had higher expression of PD-1, PD-L1, LAG-3, and TIM-3, compared with the non-responders.

Phase Ib KEYNOTE-028 trial had 20 different cohorts of previously treated patients with PD-L1-positive advanced tumors. In the anal cancer cohort, 32 (74\%) out of 43 screened patients presented PD-L1 expression $\geq 1 \%$ in the tumor cells. ${ }^{28}$ Twenty-five patients, of which $52 \%$ had been submitted to two previous lines of therapy, were submitted to pembrolizumab $10 \mathrm{mg} / \mathrm{kg}$ each two weeks. ORR was $17 \%$, and 10 patients showed stable disease, leading to a DCR of 58\%. Median PFS and OS were 3.0 months and 9.3 months, respectively. The use of pembrolizumab in SCCA patients was safe, with no unexpected toxicities. 
The phase II study KEYNOTE-158 analyzed the use of pembrolizumab in 112 previously treated patients with SCCA, irrespective of PD-L1 expression. ${ }^{14}$ In the overall population, $67 \%$ had PD-L1 expression $\geq 1 \%$ and $75 \%$ of the patients had been submitted to at least two lines of systemic therapy. ORR was $11.6 \%$, of which 6 patients reached CR. PD-L1-positive patients presented higher ORR compared to the negative counterparts: $14.7 \%$ versus $3.3 \%$, respectively. The median PFS was 2.0 months and the OS was 11.9 months.

The new humanized anti-PD-1 monoclonal antibody retifanlimab (INCMGA0012) was evaluated in the Phase 2 study POD1UM-202, with 94 patients with advanced SCCA after platinum failure, regardless of PD-L1 status. ${ }^{29}$ Interestingly, $10 \%$ of the overall population was composed of HIV-positive patients, since they had negative viral load, CD4 cells $\geq 300 / \mathrm{mm} 3$, and were on antiretroviral therapy. The ORR was $13.8 \%$, and there was no difference based on HIV or PD-L1 status. The median duration of response (DOR) was 9.5 months. The median PFS and OS were 2.3 months and 10.1 months, respectively. Despite the presence of HIV-positive patients, the safety profile was similar to that found in HIV-negative patients and to the other anti-PD-1 monoclonal antibodies.

Likewise, the safety of ICIs in the population of patients with SCCA and HIV infection was also demonstrated in the AMC 095 study. ${ }^{30}$ Nivolumab was used in 37 HIV-positive patients with advanced solid tumors and CD4 count $>100$ cells $/ \mu$ L. Five patients had SCCA. ORR was $24 \%$ and the rate of serious treatment-related adverse events (TRAEs) was $11 \%$. The viral load remained stable and negative in $97 \%$ of the patients throughout the treatment. On the other hand, there was a trend for a reduction in the $\mathrm{CD} 4$ count at 6-week interval.

\section{Adoptive T-Cell Therapies}

Adoptive T-cell therapy involving the modification of T-cell receptors (TCR) and the creation of chimeric antigen receptors (CAR) has demonstrated promising findings in SCCA. In a phase I/II study with 9 patients with advanced HPV-positive tumors, of which 4 had SCCA, patients had their T-cells extracted and modified to identify the HLA-A 2:1 epitope HPV16 E6 (E6 TCR T-cells) followed by an autologous infusion. ${ }^{31}$ Two partial responses were obtained, lasting 3 and 6 months. No doselimiting or autoimmune effects were observed.

Likewise, preliminary results of the Phase I study with modified T-cells were recently presented to identify the
HLA-A 02:01 HPV16 E7 epitope (E7 TCR T-cells) in patients with refractory HPV16-positive tumors. ${ }^{32}$ In the cohort of 12 patients, there were 2 patients with previously treated advanced SCCA. Both patients experienced partial responses lasting 3 and 9 months.

Other immunotherapeutic agents have also been evaluated in advanced SCCA. Axalimogene filolisbac (ADXS11-011) consists of a live, irreversibly attenuated and nonpathogenic strain of the intracellular bacterium Listeria monocytogenes, which is bioengineered to secrete an antigen-adjuvant fusion protein between Listeriolysin O (LLO) and the HPV-16 E7 oncoprotein. ${ }^{33}$ It may stimulate tumor-specific responses against HPV-associated cancers. In a first-in-human phase 2 study of 36 previously treated patients with advanced SCCA, ADXS11-011 induced an ORR of 3.4\% and a median 6-month PFS of $15.5 \%{ }^{33}$ In addition, grade 3 adverse events were noted in 10 patients, with the majority being cytokine-release symptoms. One grade 4 adverse event was noted, and no grade 5 adverse events occurred. Despite being safe, the primary endpoints (ORR $\geq 10 \%$ or 6-month PFS rate $\geq 20 \%$ ) were not met to proceed to the second phase of the study.

The hypothesis that anti-PD1 therapy may augment vaccine-induced immune responses was tested in a phase II trial of the combination of ISA 101, a synthetic longpeptide HPV-16 vaccine, with nivolumab in patients with incurable HPV16-positive cancers. ${ }^{34}$ There was 1 patient with SCCA in the cohort of 24 patients. ORR was $33 \%$, median PFS was 2.7 months, and median OS was not achieved in a median follow-up of 8.6 months. The treatment was safe, with one patient each presenting grade 3 transaminase and grade 4 lipase elevations. Such promising findings deserve further evaluation in a larger randomized clinical trial.

\section{Anti-EGFR Monoclonal Antibodies}

Since $R A S$ and BRAF mutations are rare in SCCA and epidermal growth factor receptor (EGFR) is commonly overexpressed in squamous tumors, anti-EGFR therapy has been intensively explored in the treatment of SCCA. The use of cetuximab and panitumumab has been described in retrospective series, either as single-agent or in combination with chemotherapy, with promising results. ${ }^{35}$ Study with 56 patients with previously treated advanced SCCA reported ORR of $41 \%$, median PFS of 4.3 months, and OS of 16.0 months. ${ }^{36}$ Cetuximab and panitumumab were used by $63 \%$ and $37 \%$ of the 
patients, respectively. In $90 \%$ of the patients, the monoclonal antibodies were used in combination with chemotherapy. Grade 3 and 4 adverse events were not reported.

The recently presented randomized phase II study CARACAS addressed the safety and efficacy of the dual PD-L1 and EGFR blockade. ${ }^{37}$ Sixty patients with refractory SCCA were randomized to avelumab alone or in combination with cetuximab. Inclusion of HIV-positive patients were allowed since they were on antiretroviral therapy and had negative viral load. ORR, the primary endpoint, was $17 \%$ in the combination arm versus $10 \%$ in the avelumab alone arm. With a median follow-up of 11 months, median PFS was 3.88 months versus 2.05 months, respectively. The most common TRAEs were fatigue in $17 \%$ of the patients in avelumab arm, and skin and subcutaneous disorders in the combination arm (87\%), but only 2 patients $(7 \%)$ permanently interrupted the treatment due to TRAE.

\section{Antiangiogenics}

Angiogenesis mediated by vascular endothelial growth factor (VEGF) is associated with immune evasion mechanisms and an immunosuppressive tumor microenvironment. The combination of immunotherapy with antiangiogenics has been successful in the treatment of hepatocellular carcinoma, and it was recently evaluated in a phase II study with 20 previously treated patients with advanced SCCA. ${ }^{38}$ The combination of atezolizumab and bevacizumab reached an ORR of $11 \%$, with 11 additional patients presenting stable disease. With a median follow-up of 9.6 months, median PFS and OS were 4.1 and 11.6 months, respectively. However, the rate of grade 3 and 4 adverse events was $35 \%$, and 1 patient died due to intestinal perforation.

\section{Others (Miscellanea)}

Other strategies have been explored, such as PEN-866, which is a miniature drug conjugate that links a heat shock protein 90 (HSP90) binding small molecule to a SN-38 cytotoxic payload. HSP90 is highly expressed in advanced malignancies. ${ }^{39}$ PEN-866 targets and binds to activated tumor HSP90 protein, releases its cytotoxic payload, and may result in tumor regression. The first-inhuman phase I study evaluated 30 patients with advanced refractory solid malignancies. The only one responder was a patient with SCCA, but six additional patients presented decrease of the target lesions. The most frequent $(\geq 20 \%)$ adverse events were nausea (50\%), fatigue (43\%), and diarrhea $(40 \%) .{ }^{39}$

\section{Perspectives}

The latest advances in the systemic therapy of SCCA have provided the framework for the conception of new clinical trials (Table 1). Therefore, carboplatin plus paclitaxel and mDCF have become the backbone for novel agents. Promising findings derived from the studies with ICIs in refractory disease have prompted their evaluation in firstline setting and in locally advanced disease. Anti-PD-1 monoclonal antibodies, such as retifanlimab and nivolumab, have been studied in phase III trials evaluating the combination with carboplatin plus paclitaxel in first-line therapy. Atezolizumab is also being studied in combination with the triplet $\mathrm{mDCF}$ in advanced disease. Ongoing randomized clinical trials are investigating the use of ICIs in locally advanced disease, either in combination with chemotherapy or as consolidation therapy after chemoradiation. In refractory disease, the combination of ipilimumab plus nivolumab has also been explored in a randomized phase II study. Novel technologies, such as XmAb20717, a bispecific antibody that simultaneously targets the immune checkpoint receptors PD-1 and CTLA-4, are being investigated in phase I studies with SCCA patients, as well as other immunotherapeutic approaches (Table 1). Based on the ongoing clinical trials evaluating new agents in SCCA, it is possible that immunotherapy begins to play a larger role in the therapeutic management of this challenging disease in the next few years.

\section{Conclusions}

SCCA is an HPV-related malignancy with rising incidence in the past few years, characterized by high rates of complete response to chemoradiotherapy. However, a meaningful subgroup of patients with locally advanced disease presents disease recurrence in long-term followup, demanding treatments with high morbidity and important impact in the quality of life. In metastatic or unresectable disease, palliative chemotherapy may be used, but it is still associated with a dismal prognosis. Novel agents are urgently needed in the systemic therapy of SCCA. Immunotherapeutic approaches are the main therapeutic strategy being investigated in anal cancer, either with immune checkpoint inhibitors or with vaccines and adoptive T-cell therapies. Combination of immunotherapy with anti-EGFR monoclonal antibodies 
Table I Ongoing Clinical Trials Evaluating Systemic Therapy in Anal Cancer*

\begin{tabular}{|c|c|c|c|c|}
\hline Population & Intervention & Control Arm & $\begin{array}{l}\text { Other Tumors } \\
\text { Included }\end{array}$ & $\begin{array}{c}\text { ClinicalTrials.gov } \\
\text { Identifier }\end{array}$ \\
\hline \multicolumn{5}{|l|}{ Phase III } \\
\hline $\begin{array}{l}\text { Locally advanced or } \\
\text { Metastatic }\end{array}$ & $\begin{array}{c}\text { Carboplatin + Paclitaxel + } \\
\text { Retifanlimab }\end{array}$ & $\begin{array}{c}\text { Carboplatin + Paclitaxel + } \\
\text { Placebo }\end{array}$ & No & NCT04472429 \\
\hline Metastatic & $\begin{array}{c}\text { Carboplatin + Paclitaxel + } \\
\text { Nivolumab }\end{array}$ & Carboplatin plus Paclitaxel & No & NCT0444492I \\
\hline Advanced & Anti-PD-I/PD-LI I year & $\begin{array}{l}\text { Anti-PD- I/PD-LI until disease } \\
\text { progression }\end{array}$ & Yes & NCT04I57985 \\
\hline $\begin{array}{l}\text { High risk stage II-IIIB (after } \\
\text { treatment) }\end{array}$ & Nivolumab & No & No & NCT032337II \\
\hline \multicolumn{5}{|l|}{ Randomized phase II } \\
\hline Locally advanced & $\begin{array}{c}\text { Durvalumab + Mitomycin + } \\
\text { 5FU with RT }\end{array}$ & Mitomycin + 5FU with RT & No & NCT04230759 \\
\hline Metastatic & Ipilimumab + Nivolumab & Nivolumab & No & NCT023I4I69 \\
\hline Metastatic or unresectable & $\mathrm{mDCF}+$ Atezolizumab & $\mathrm{mDCF}$ & No & NCT035I9295 \\
\hline \multicolumn{5}{|l|}{ Single-arm phase II } \\
\hline Advanced & Avelumab + Valproic acid & - & Yes & NCT03357757 \\
\hline Metastatic & Pembrolizumab & - & No & NCT02919969 \\
\hline Metastatic or unresectable & Pembrolizumab & - & Yes & NCT02628067 \\
\hline \multicolumn{5}{|l|}{ Phase I } \\
\hline Metastatic/recurrent & PDSOI0I + M7824 + NHS-ILI2 & - & Yes & NCT04287868 \\
\hline Advanced & XmAb207I7 & - & Yes & NCT035I7488 \\
\hline Advanced & Ipilimumab + Nivolumab & - & Yes & NCT0240886I \\
\hline
\end{tabular}

Note: *Active and recruiting on November 28, 2020.

Abbreviations: PD-I, programmed cell death-I; PD-LI, programmed cell death-I-ligand; 5FU, 5-fluorouracil; RT, radiation therapy; mDCF, modified docetaxel, cisplatin, 5-fluorouracil.

or with antiangiogenics have also been evaluated in clinical trials, which may bring practice-changing advances in the systemic therapy of SCCA in the next few years.

\section{Disclosure}

The authors report no conflicts of interest in this work.

\section{References}

1. Siegel RL, Miller KD, Jemal A. Cancer statistics, 2020. CA Cancer J Clin. 2020;70(1):7-30. doi:10.3322/caac.21590

2. Siegel RL, Miller KD, Jemal A. Cancer statistics, 2018. CA Cancer J Clin. 2018;68(1):7-30. doi:10.3322/caac. 21442
3. Siegel RL, Miller KD, Jemal A. Cancer statistics, 2019. CA Cancer J Clin. 2019;69(1):7-34. doi:10.3322/caac.21551

4. Islami F, Ferlay J, Lortet-Tieulent J, Bray F, Jemal A. International trends in anal cancer incidence rates. Int J Epidemiol. 2017;46 (3):924-938. doi:10.1093/ije/dyw276

5. James RD, Glynne-Jones R, Meadows HM, et al. Mitomycin or cisplatin chemoradiation with or without maintenance chemotherapy for treatment of squamous-cell carcinoma of the anus (ACT II): a randomised, Phase 3, open-label, $2 \times 2$ factorial trial. Lancet Oncol. 2013;14(6):516-524. doi:10.1016/S1470-2045(13)70086-X

6. Ajani JA, Winter KA, Gunderson LL, et al. Fluorouracil, mitomycin, and radiotherapy vs fluorouracil, cisplatin, and radiotherapy for carcinoma of the anal canal: a randomized controlled trial. JAMA. 2008;299(16):1914-1921. doi:10.1001/jama.299.16.1914

7. Nigro ND, Vaitkevicius VK, Considine B. Combined therapy for cancer of the anal canal: a preliminary report. Dis Colon Rectum. 1974;17(3):354-356. doi:10.1007/BF02586980 
8. Peiffert D, Tournier-Rangeard L, Gérard JP, et al. Induction chemotherapy and dose intensification of the radiation boost in locally advanced anal canal carcinoma: final analysis of the randomized UNICANCER ACCORD 03 trial. $J$ Clin Oncol. 2012;30 (16):1941-1948. doi:10.1200/JCO.2011.35.4837

9. Glynne-Jones R, Sebag-Montefiore D, Meadows HM, et al. Best time to assess complete clinical response after chemoradiotherapy in squamous cell carcinoma of the anus (ACT II): a post-hoc analysis of randomised controlled phase 3 trial. Lancet Oncol. 2017;18 (3):347-356. doi:10.1016/S1470-2045(17)30071-2

10. Northover J, Glynne-Jones R, Sebag-Montefiore D, et al. Chemoradiation for the treatment of epidermoid anal cancer: 13-year follow-up of the first randomised UKCCCR Anal Cancer Trial (ACT I). Br J Cancer. 2010;102(7):1123-1128. doi:10.1038/sj.bjc.6605605

11. Gunderson LL, Winter KA, Ajani JA, et al. Long-term update of US GI intergroup RTOG 98-11 phase III trial for anal carcinoma: survival, relapse, and colostomy failure with concurrent chemoradiation involving fluorouracil/mitomycin versus fluorouracil/cisplatin. J Clin Oncol. 2012;30(35):4344-4351. doi:10.1200/JCO.2012.43.8085

12. Rao S, Sclafani F, Eng C, et al. International rare cancers initiative multicenter randomized phase ii trial of cisplatin and fluorouracil versus carboplatin and paclitaxel in advanced anal cancer: interAAct. $J$ Clin Oncol. 2020:JCO1903266. doi:10.1200/ JCO.19.03266

13. Morris VK, Salem ME, Nimeiri H, et al. Nivolumab for previously treated unresectable metastatic anal cancer (NCI9673): a multicentre, single-arm, phase 2 study. Lancet Oncol. 2017;18(4):446-453. doi:10.1016/S1470-2045(17)30104-3

14. Marabelle A, Cassier PA, Fakih M, et al. Pembrolizumab for advanced anal squamous cell carcinoma (ASCC): results from the multicohort, phase II KEYNOTE-158 study. Am Soc Clin Oncol. 2020.

15. Benson AB, Venook AP, Al-Hawary MM, et al. Anal carcinoma, version 2.2018, NCCN clinical practice guidelines in oncology. J Nat Comprehen Cancer Network. 2018;16(7):852-871. doi:10.6004/ jnccn.2018.0060

16. Brown S, Skinner P, Tidy J, Smith J, Sharp F, Hosie K. Outcome after surgical resection for high-grade anal intraepithelial neoplasia (Bowen's disease). Br j Surg. 1999;86(8):1063-1066. doi:10.1046/ j.1365-2168.1999.01184.x

17. Kim S, François E, André T, et al. Docetaxel, cisplatin, and fluorouracil chemotherapy for metastatic or unresectable locally recurrent anal squamous cell carcinoma (Epitopes-HPV02): a multicentre, single-arm, phase 2 study. Lancet Oncol. 2018;19(8):1094-1106. doi:10.1016/S1470-2045(18)30321-8

18. Jacome AA, Eng C. Experimental and investigational drugs for the treatment of anal cancer. Expert Opin Investig Drugs. 2018;27 (12):941-950. doi:10.1080/13543784.2018.1543659

19. Chung JH, Sanford E, Johnson A, et al. Comprehensive genomic profiling of anal squamous cell carcinoma reveals distinct genomically defined classes. Ann Oncol. 2016;27(7):1336-1341. doi:10.1093/annonc/mdw152

20. Morris V, Rao X, Pickering C, et al. Comprehensive genomic profiling of metastatic squamous cell carcinoma of the anal canal. Mol Cancer Res. 2017;15(11):1542-1550. doi:10.1158/1541-7786.MCR17-0060

21. Siegel EM, Eschrich S, Winter K, et al. Epigenomic characterization of locally advanced anal cancer: a radiation therapy oncology group 98-11 specimen study. Dis Colon Rectum. 2014;57(8):941-957. doi:10.1097/DCR.0000000000000160

22. Smaglo BG, Tesfaye A, Halfdanarson TR, et al. Comprehensive multiplatform biomarker analysis of 199 anal squamous cell carcinomas. Oncotarget. 2015;6(41):43594-43604. doi:10.18632/oncotarget.6202

23. Phuong L, Rajdev L. Immunotherapy in Anal Cancer. Curr Oncol Rep. 2020;22(9):1-6. doi:10.1007/s11912-020-00946-3
24. Jiang K, Martens B, Meyer L, Truong K, Lauwers GY. A mismatch repair-deficient and HPV-negative anorectal squamous cell carcinoma. Virchows Archiv. 2019;474(6):769-773. doi:10.1007/ s00428-019-02530-y

25. Smola S. Immunopathogenesis of HPV-Associated Cancers and Prospects for Immunotherapy. Viruses. 2017;9(9):254. doi:10.3390/v9090254

26. Heusinkveld M, de Vos van Steenwijk PJ, Goedemans R, et al. M2 macrophages induced by prostaglandin E2 and IL-6 from cervical carcinoma are switched to activated M1 macrophages by CD4+ Th1 cells. J Immunol. 2011;187(3):1157-1165. doi:10.4049/jimmunol.1100889

27. Spehner L, Kim S, Vienot A, et al. Anti-telomerase CD4+ Th1 immunity and monocytic-myeloid-derived-suppressor cells are associated with long-term efficacy achieved by docetaxel, cisplatin, and 5-fluorouracil (DCF) in advanced anal squamous cell carcinoma: translational study of epitopes-HPV01 and 02 trials. Int J Mol Sci. 2020;21(18):6838.

28. Ott PA, Piha-Paul SA, Munster P, et al. Safety and antitumor activity of the anti-PD-1 antibody pembrolizumab in patients with recurrent carcinoma of the anal canal. Ann Oncol. 2017;28(5):1036-1041. doi:10.1093/annonc/mdx029

29. Rao S, Capdevila J, Gilbert D, et al. LBA42 POD1UM-202: phase II study of retifanlimab in patients (pts) with squamous carcinoma of the anal canal (SCAC) who progressed following platinum-based chemotherapy. Ann Oncol. 2020;31:S1170-S1171. doi:10.1016/j. annonc.2020.08.2272

30. Rajdev L, Lensing S, Ramos J, et al. 1023MO AMC 095: a report of nivolumab (nivo) in advanced HIV associated solid tumours (ST). Ann Oncol. 2020;31:S706. doi:10.1016/j.annonc.2020.08.1143

31. Hinrichs CS, Doran SL, Stevanovic S, et al. A phase I/II clinical trial of E6 T-cell receptor gene therapy for human papillomavirus (HPV)-associated epithelial cancers. Am Soc Clin Oncol. 2017;35 (15_suppl):3009. doi:10.1200/JCO.2017.35.15_suppl.3009

32. Norberg S, Nagarsheth N, Sinkoe A, et al. Safety and clinical activity of gene-engineered T-cell therapy targeting HPV-16 E7 for epithelial cancers. Am Soc Clin Oncol. 2020;38(15_suppl):101. doi:10.1200/ JCO.2020.38.15 suppl.101

33. Eng C, Fakih M, Amin M, et al. A phase II study of axalimogene filolisbac for patients with previously treated, unresectable, persistent/recurrent loco-regional or metastatic anal cancer. Oncotarget. 2020;11(15):1334-1343. doi:10.18632/oncotarget.27536

34. Glisson B, Massarelli E, William W, et al. 1136ONivolumab and ISA $101 \mathrm{HPV}$ vaccine in incurable HPV-16+ cancer. Ann Oncol. 2017;28 (suppl_5):v403-v404. doi:10.1093/annonc/mdx376.002

35. Morris V, Eng C. Metastatic anal cancer and novel agents. Surgical Oncol Clin. 2017;26(1):133-142. doi:10.1016/j.soc.2016.07.008

36. Rogers JE, Jácome AA, Ohinata A, et al. Outcomes with anti-EGFR monoclonal antibodies in metastatic and recurrent anal squamous cell carcinoma. Expert Rev Anticancer Ther. 2020;20(10):901-908. doi:10.1080/14737140.2020.1810573

37. Lonardi S, Pietrantonio F, Prete A, et al. Final results of the CARACAS study: randomized phase II trial of avelumab alone or with cetuximab for unresectable, locally advanced or metastatic squamous cell anal carcinoma progressed to at least one line of treatment. Ann Oncol. 2020;31:S412. doi:10.1016/j.annonc.2020.08.513

38. Morris V, Liu S, Johnson B, et al. Atezolizumab in combination with bevacizumab for patients with unresectable/metastatic anal cancer. Ann Oncol. 2020;31:S412. doi:10.1016/j.annonc.2020.08.514

39. Falchook GS, Bendell JC, Ulahannan SV, et al. Pen-866, a miniature drug conjugate of a heat shock protein 90 (HSP90) ligand linked to SN38 for patients with advanced solid malignancies: phase I and expansion cohort results. Am Soc Clin Oncol. 2020;38 (15_suppl):3515. doi:10.1200/JCO.2020.38.15_suppl.3515 


\section{Publish your work in this journal}

The Journal of Experimental Pharmacology is an international, peerreviewed, open access journal publishing original research, reports, reviews and commentaries on all areas of laboratory and experimental pharmacology. The manuscript management system is completely

online and includes a very quick and fair peer-review system. Visit http://www.dovepress.com/testimonials.php to read real quotes from published authors. 